\title{
Neuer Vorstand im EbM-Netzwerk
}

Prof. Dr. Andreas Sönnichsen (Medizinische Universität Wien) ist der neue Vorsitzende des Deutschen Netzwerks Evidenzbasierte Medizin. Ihm zur Seite stehen Dr. Dagmar Lühmann (Universitätsklinikum Hamburg-Eppendorf) und PD Dr. Nicole Skoetz (Universitätsklinikum Köln) als Stellvertreterinnen sowie Prof. Dr. Gabriele Meyer (Martin-Luther-Universität Halle-Wittenberg) als Schriftführerin.

Auf der Mitgliederversammlung des Deutschen Netzwerks Evidenzbasierte Medizin am 21. März 2019 im Rahmen der 20. Jahrestagung in Berlin wurde Prof. Dr. med. Andreas Sönnichsen zum neuen Ersten Vorsitzenden gewählt. Er folgt damit Dr. med. Dagmar Lühmann, Institut für Allgemeinmedizin am Zentrum für Psychosoziale Medizin im Universitätsklinikum Hamburg-Eppendorf, die nach zweijähriger Amtszeit in die Position der Ersten Stellvertretenden Vorsitzenden wechselt. PD Dr. med. Nicole Skoetz, Universitätsklinikum Köln und dort u.a. Leiterin der Cochrane Haematological Malignancies Group, wurde zur neuen Zweiten Stellvertretenden Vorsitzenden gewählt. Prof. Dr. Gabriele Meyer, Leiterin des Instituts für Gesundheits- und Pflegewissenschaft an der Martin-Luther-Universität HalleWittenberg, wurde erneut als schriftführendes Vorstandsmitglied des Netzwerks gewählt.

Andreas Sönnichsen leitet seit 2018 die Abteilung für Allgemein- und Familienmedizin am Zentrum für Public Health der Medizinischen Universität Wien. Zuvor war er Lehrstuhlinhaber für Allgemeinmedizin und Familienmedizin an der Universität Witten-Herdecke und zwischen 2006 und 2012 Vorstand des Instituts für Allgemein-, Familien- und Präventivmedizin der Paracelsus Medizinische Privatuniversität Salzburg.

Seit 2008 ist Sönnichsen Mitglied des EbM-Netzwerks und seit 2017 war er Erster stellvertretender Vorsitzender im geschäftsführenden Vorstand.

Als Beisitzer/in im Vorstand wurden gewählt:

- Udo Ehrmann, Bundesverband Prostatakrebs Selbsthilfe e.V., Bremen

- Dr. med. Lars G. Hemkens, ceb - Basel Institut für Klinische Epidemiologie \& Biostatistik, Universitätsspital Basel

- Dimitra Panteli, Ärztin, MScPH, DrPH, Technische Universität Berlin, Fachgebiet Management im Gesundheitswesen

- Prof. Dr. med. Stefan Sauerland, Institut für Qualität und Wirtschaftlichkeit im Gesundheitswesen (IQWiG), Köln

Der neue Vorstand dankte der Mitgliederversammlung für das Vertrauen und dem ehemaligen Vorstandsmitglied, Prof. Dr. med. Ingrid Mühlhauser, und der Beisitzerin im Vorstand, Dr. med. Ulrike Euler, für die geleistete Arbeit.

Das EbM-Netzwerk setzt sich dafür ein, dass alle Bürgerinnen und Bürger eine gesundheitliche Versorgung erhalten, die auf bester wissenschaftlicher Erkenntnis und informierter Entscheidung beruht. In ihm haben sich Wissenschaftler/innen aus medizinischen, pflege- und gesundheitswissenschaftlichen Fakultäten, praktizierende Ärzte/Ärztinnen und sowie Vertreter anderer Gesundheitsberufe zusammengeschlossen (www.ebm-netzwerk.de). 